\title{
Retracer les temporalités d'une forêt emmurée
}

Le Domaine national de Chambord, les limites d'un exemple bien documenté

Tracing the temporalities of a walled forest

The national property of Chambord, the limits of a well-documented example

\section{Amélie Robert et Sylvie Servain}

\section{(2) OpenEdition}

\section{Journals}

Édition électronique

URL : http://journals.openedition.org/developpementdurable/12163

DOI : 10.4000/developpementdurable.12163

ISSN : 1772-9971

Éditeur

Association DD\&T

Référence électronique

Amélie Robert et Sylvie Servain, "Retracer les temporalités d'une forêt emmurée », Développement durable et territoires [En ligne], Vol. 9, n² I Juin 2018, mis en ligne le 15 juin 2018, consulté le 02 mai 2019. URL : http://journals.openedition.org/developpementdurable/12163 ; DOI : 10.4000/ developpementdurable.12163

Ce document a été généré automatiquement le 2 mai 2019.

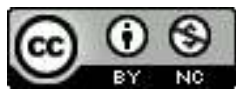

Développement Durable et Territoires est mis à disposition selon les termes de la licence Creative Commons Attribution - Pas d'Utilisation Commerciale 4.0 International. 


\title{
Retracer les temporalités d'une forêt emmurée
}

\author{
Le Domaine national de Chambord, les limites d'un exemple bien \\ documenté \\ Tracing the temporalities of a walled forest \\ The national property of Chambord, the limits of a well-documented example
}

Amélie Robert et Sylvie Servain

Les auteurs tiennent à remercier les relecteurs qui, par la pertinence de leurs remarques, ont contribué à la qualité de cet article. Ils souhaitent aussi témoigner leur gratitude au Domaine national de Chambord et son personnel pour l'accueil, le temps accordé et les documents mis à disposition, ainsi qu'à la Région Centre-Val de Loire, qui a financé les recherches dont est issu cet article.

Chambord fait nécessairement référence au château de la Renaissance auquel il faut ajouter un domaine national de 5440 hectares qui inclut un village, des fermes et surtout une forêt couvrant une grande partie de ce territoire. Le domaine apparaît représentatif de la Grande Sologne aux marges de laquelle il se situe, en raison de la prédominance de la forêt, mais aussi parce qu'il est clos. Cette situation est courante dans la région, où les engrillagements se sont multipliés au point que l'on parle de "solognisation " pour évoquer ce phénomène (Carrelet-Baltzinger, 2016). L'objectif de ces clôtures est de délimiter les propriétés et surtout, de privatiser la chasse, fonction que remplit le mur du Domaine national de Chambord ${ }^{1}$. Et pour cause, ce domaine est, dès sa création au $\mathrm{XVI}^{\mathrm{e}}$ siècle, un territoire dédié aux activités cynégétiques, ce qui n'est pas sans poser de problème pour la gestion forestière, dans la mesure où, en forte concentration, le gibier engendre des dégâts (Thoreau, 1975 ; Côté et al., 2004) - même si les effets peuvent aussi être plus positifs (Mårell, Pellerin et Rocquencourt, 2012 ; Baltzinger, 2016). Mais la forêt était-elle aussi présente à cette époque qu'aujourd'hui et quelles évolutions a-t-elle connues par la suite? C'est la question que nous nous sommes posée dans le cadre d'un projet de recherche interdisciplinaire consacré au Domaine national de Chambord et dont les objectifs principaux sont d'améliorer la compréhension des paysages et de la 
biodiversité, afin de proposer des adaptions pour la gestion de fortes populations d'ongulés sauvages et de leurs habitats ${ }^{2}$. Il s'agissait donc de retracer les dynamiques forestières sur un territoire bien délimité, puisque clos. L'approche diachronique régressive a été privilégiée, ce qui impliquait de dresser différents états des lieux, à des dates données selon les sources retenues, en vue de les comparer, en remontant le passé à partir de la situation actuelle, pour retracer les dynamiques et trajectoires forestières. Cette forêt emmurée est fort bien documentée: des sources cartographiques, iconographiques, écrites et même, pour la période plus récente, des bases de données sont disponibles et renseignent sur l'occupation des sols et les paysages à différentes dates. Pour autant, nous montrerons ici que, même pour un tel espace, les limites inhérentes aux sources contraignent l'analyse des temporalités par leur qualité, leur fiabilité, leur disponibilité et leur niveau d'information. Les sources étant nombreuses et variées pour notre espace d'étude, nous nous sommes focalisées sur les données cartographiques et iconographiques. Nous verrons dans un premier temps quelles sont les dates clés et les sources sélectionnées pour dresser des états des lieux de cette forêt, en précisant les raisons qui ont motivé notre choix. Puis, nous présenterons les implications de la confrontation de ce choix avec la réalité des sources, en termes de disponibilité, de fiabilité et de pertinence, en distinguant deux périodes, avant et après 1949 , date charnière retenue au regard des sources, des temporalités du domaine et du projet. Enfin, nous nous intéresserons aux implications de leur analyse, par SIG, et aux possibilités de faire appel à d'autres sources.

\section{Temporalités et dates clés : choix théorique et confrontation des sources disponibles}

2 La première étape comprend deux phases : le choix des temporalités et des dates clés, où des états des lieux de l'occupation des sols seront dressés, puis l'inventaire des sources existantes.

\subsection{Le choix théorique des temporalités et des dates clés}

3 Étudier les dynamiques de la forêt suppose d'effectuer des choix de temporalités à considérer, mais lesquelles faut-il privilégier? Celles des sylvosystèmes qui s'inscrivent dans les temps longs de l'environnement ou celles plus brèves des sociétés (ou sociosystèmes) qui, par leurs pratiques et leurs usages, marquent leurs empreintes sur ces forêts?

4 «Le temps long est à la fois celui de la stabilité relative des paysages et des cycles séculaires des échanges. Le temps "social" ou "institutionnel" de moyen terme est à la fois culturel et politique, structurel et événementiel, collectif et pluriel » (Dubar et Rolle, 2008).

5 De là découle le choix du type de sources et de la période étudiée et, par là même, de la date la plus ancienne qui sera considérée. Dans le cas de notre étude, il s'agit de retracer les dynamiques forestières au sein du Domaine national de Chambord en lien avec les pratiques de chasse et les populations d'ongulés sauvages, afin de comprendre comment se sont construits les paysages actuels et d'identifier la rémanence des usages passés ${ }^{3}$. En raison d'une plus grande disponibilité des données, la période choisie pour l'étude des ongulés sauvages s'étend de 1950 à aujourd'hui. Nous nous focalisons donc sur celle-ci. 
Toutefois, pour comprendre les dynamiques récentes des paysages, il est nécessaire de considérer une période plus longue, pour mieux caractériser les trajectoires évolutives des dynamiques spontanées de la végétation, s'inscrivant dans le temps long. L'approche relève de la biogéographie historique, celle qui «se fonde sur la dimension historique pour offrir une clé d'interprétation supplémentaire dans la compréhension des forêts, résultant d'une interaction pluriséculaire entre les sociétés et leur milieu » (Galochet et Glon, 2010). Ainsi, l'analyse depuis la création du domaine, au XVI e siècle, permet de prendre en compte de manière croisée les dynamiques d'origine externe, impulsées par la société, autant que les dynamiques d'origine interne, qui sont celles spontanées - guidées par le fonctionnement biologique - de la forêt ${ }^{4}$.

Une fois ce choix de la période effectué, il importe de sélectionner les dates auxquelles les états des lieux seront dressés. Elles peuvent être réparties de manière régulière dans le temps ou choisies en fonction d'événements qui ont pu influencer les dynamiques forestières, via des modifications de pratiques. La première option est plus aisément envisageable sur des temps courts, surtout récents - notamment pour des raisons de disponibilité de sources, question que nous aborderons ultérieurement. Ainsi, pour leur identification des dynamiques agricoles au cours des cinquante dernières années, T. Houet et al. (2008) ont pu se fonder sur des états des lieux décennaux, auxquels ils ont même associé un suivi annuel sur un temps plus court encore (les sept dernières années). Mais un tel suivi ne peut être réalisé sur plusieurs siècles, d'une part parce que l'analyse des données serait trop chronophage et sans pertinence réelle dans notre cas, au regard des dynamiques étudiées, en l'occurrence forestières, et d'autre part parce que, nous le verrons, des données à des pas de temps aussi rapprochés ne sont pas toujours disponibles. Or, des états des lieux trop espacés peuvent amener à négliger certaines mutations : une baisse de la superficie forestière suivie d'une hausse de même ampleur pourrait être considérée comme une stagnation si seuls deux états des lieux sont dressés, avant la baisse et après la hausse. Il apparaît donc plus judicieux de prendre en compte les temporalités de la société, l'évolution des pratiques anthropiques, afin de choisir des dates marquant des changements pour dresser les états des lieux.

Dans le cas du Domaine national de Chambord, les sources scripturales sont nombreuses et permettent ainsi de connaître de manière détaillée les pratiques et leurs évolutions, d'autant plus qu'une histoire du domaine, fondée sur des données d'archives, a été retracée par l'ancien chef de son service forestier (Thoreau, 1975). De l'analyse de ces sources, il ressort plusieurs faits, se rapportant à la création, puis à la gestion du domaine, aux dispositifs de protection qui y sont appliqués ou aux pratiques sylvicoles, en l'occurrence les plantations forestières (figure 1). Ces événements doivent être identifiés, mais, pour comprendre les dynamiques de l'occupation des sols, tous ne se situent pas sur le même plan et, dès lors, tous ne doivent pas être obligatoirement considérés. Certains se distinguent comme étant particulièrement marquants et susceptibles d'avoir eu un impact sur les paysages. Leurs dates sont donc jugées a priori pertinentes pour dresser des états des lieux de l'occupation des sols. Il s'agit notamment :

- du début de la construction du mur entourant le domaine en 1524 ;

- des reboisements, en 1821 pour les premiers, puis de 1945 à 1955 ;

- du classement du domaine en Réserve nationale de chasse et de faune sauvage et de sa soumission au régime forestier en 1947 ;

- de la création d'un commissariat à l'aménagement par le président Pompidou (première tentative de coordination de l'administration du domaine, pour l'organisation des chasses 
présidentielles) en 1970 ;

- du changement de statut du domaine, devenant EPIC (Établissement public industriel et commercial) en 2005.

8 Ces dates correspondent certes à des faits marquants dans la gestion du domaine et notamment de ses forêts, mais pas nécessairement à des changements paysagers. Par ailleurs, il faut aussi considérer qu'il n'y a pas toujours adéquation entre la date d'un événement et celle à partir de laquelle ses effets sont perceptibles. En effet, certains faits nécessitent un temps de latence qu'il n'est pas toujours aisé d'estimer. Dans le cas de reboisements, un délai est nécessaire entre le moment où la plantation est réalisée et celui où la formation végétale pourra être considérée comme une forêt. Plus généralement, les dynamiques spontanées à l'œuvre s'inscrivent dans la durée. Mais, de même, un changement de statut ou de gestion d'un domaine forestier n'aura pas nécessairement un impact immédiat, si impact il y a : la prise de décisions, l'influence de celles-ci sur les pratiques prennent du temps et les changements peuvent être progressifs. Le choix des dates clés, auxquelles des états des lieux seront dressés, s'avère ainsi complexe même s'il n'est encore que théorique. Vient ensuite la confrontation avec la réalité des sources et très vite se pose la question de leur disponibilité.

Figure 1. Le Domaine national de Chambord entre 1500 et aujourd'hui : événements susceptibles de marquer les paysages et choix de dates jugées clés (encadrées en rouge)

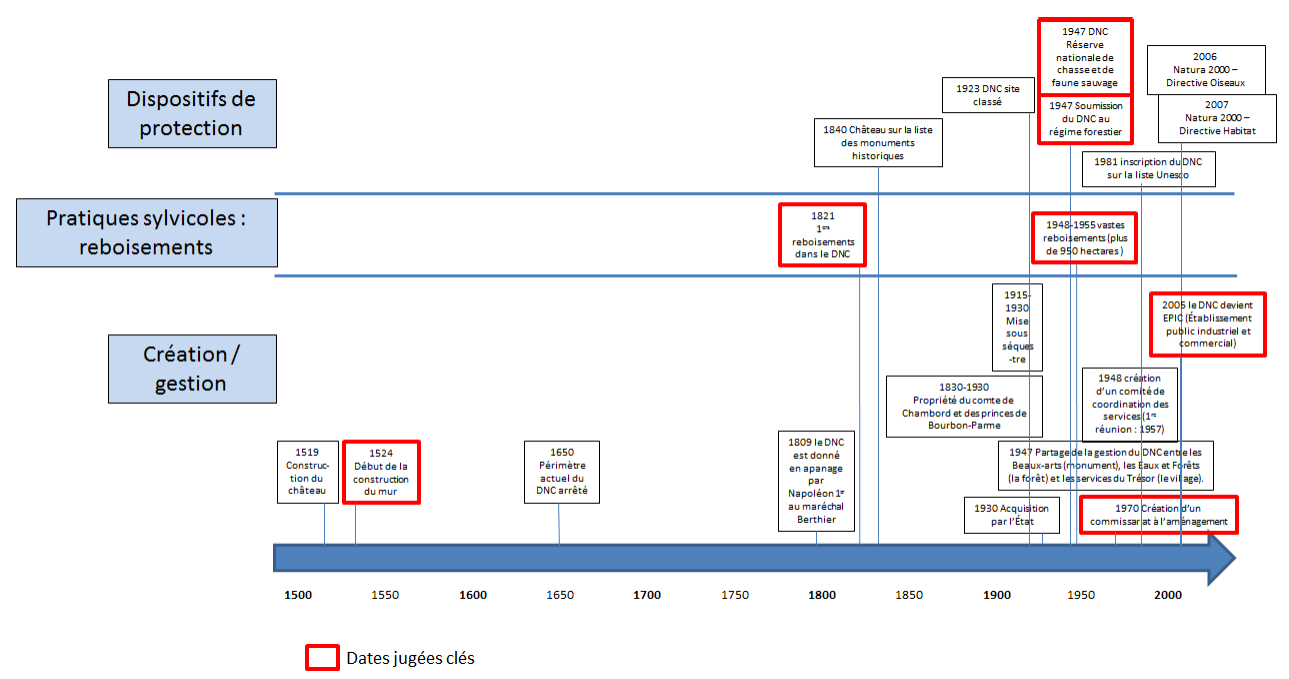

DNC : Domaine national de Chambord

\subsection{Inventaire des sources et focalisation sur les données spatialisées}

9 Pour connaître l'occupation des sols et, par là même, identifier les dynamiques paysagères, plusieurs sources sont mobilisables. Lesquelles faut-il privilégier et lesquelles sont disponibles dans le cas du Domaine national de Chambord?

Les photographies aériennes sont traditionnellement les plus utilisées par les géographes, éventuellement complétées par des images satellitaires, pour connaître les dynamiques récentes de l'occupation des sols (Amat et Hotyat, 1980 ; Amat, 1987 ; Houet et al., 2008 ; Robert, 2011 ; Servain-Courant et Yengué, 2011 ; Pin, Rode, Servain, 2013). Pour les états des lieux les plus anciens, elles ne sont pas disponibles, mais, pour autant, les sources ne 
se limitent pas aux cartes. Peuvent s'ajouter des plans, des textes, des statistiques anciennes et les cadastres qui renseignent aussi sur les paysages (Jacob, 2010; Franchomme et Dubois, 2010). Mais la mobilisation de ces différentes sources est alors fonction des méthodes retenues (Valette et Carozza, 2010) et, derrière cela, des objectifs fixés et des disciplines :

11 «Plusieurs types de méthodes existent pour mesurer les changements du paysage. Si on définit le paysage comme une portion d'espace vue à partir d'un point situé à la surface de la Terre ou proche de celle-ci, on peut comparer des photographies diachroniques (Lepart et al., 1996 [5] ; Debussche et al., $\left.\left.1999{ }^{[6}\right]\right)$. Les autres techniques de mesure des changements comparent et analysent des documents de type cartographique (cadastres, cartes anciennes) et sont plus appropriées à une analyse du paysage tel qu'il est défini en écologie du paysage [...]. Enfin, même spatialement peu explicites, les statistiques d'utilisation des sols rassemblées par recensements permettent de quantifier rapidement les changements intervenus dans l'occupation des sols » (Lepart et al., 2007).

D'autres sources peuvent être ajoutées :

- scripturales, mobilisées par certains géographes - X. Rochel (2013) se fonde ainsi sur les registres de martelages pour connaître les forêts du XVIII ${ }^{\mathrm{e}}$ siècle ;

- iconographiques autres que les photographies aériennes et données spatiales - ce qui inclut les photographies prises au sol, les cartes postales, les tableaux d'époque (Amat, 2015);

- ou même les sources orales comme les récits et témoignages ;

- voire un mélange de ces différentes sources, pour approcher - ou tenter d'approcher les paysages passés, pour lesquels les sources sont lacunaires, et par là même leurs dynamiques (Robert, 2011).

Il s'agit là d' " archives humaines », mais on peut aussi envisager de les compléter par des « archives naturelles (palynologie, anthracologie) » (Rochel, 2013), ce qui inclut également de nouvelles méthodes comme l'analyse des matières organiques du sol par la spectroscopie proche infrarouge (Ertlen et Schwartz, 2010). Les données géoarchéologiques et paléoécologiques "prennent [en fait] le relais » des données historiques en deçà du XVIII siècle (Ertlen et Schwartz, 2010). Enfin, les images LiDAR offrent de nouvelles perspectives pour reconstituer les paysages du passé (Georges-Leroy et al., 2011 ; Horen et al., 2015 ; de Matos Machado et al., 2016).

Dans le cas de cette étude, nous nous limiterons aux "archives humaines » spatialisées que sont les cartes et les plans, parce qu'elles sont directement intégrables dans un SIG et que notre objectif est de cartographier les dynamiques de l'occupation des sols, tout en les complétant ponctuellement par des références à des sources iconographiques (gravures, peintures, fresques, photographies : voir infra). En effet, « les archives cartographiques sont a priori les meilleures pour répondre à la question de la localisation » (Ertlen et Schwartz, 2010). Dans le cas du Domaine national de Chambord, un grand nombre de sources spatialisées existent (tableau 1), mais, comme souvent, elles deviennent de plus en plus lacunaires à mesure qu'on remonte le passé (Robert, 2010) - même si, ponctuellement dans l'histoire, des exceptions existent, avec une plus grande richesse des données (voir infra) ${ }^{7}$. Elles sont aussi moins diversifiées, puisqu'il s'agit de cartes, de plans, éventuellement de gravures. Pour les états des lieux récents s'ajoutent des photographies aériennes, surtout à partir des années 1950 (même si la première photographie aérienne date de la toute fin du XIX siècle, début $\mathrm{du} \mathrm{xx}^{\mathrm{e}}$ siècle, avec l'aviation militaire), des images satellitaires (non considérées ici) à partir de 1972 ainsi que des bases de données au format vectoriel depuis 
les années 1990 (BD Topo de l'IGN par exemple). Pour le Domaine national de Chambord, il existe également deux bases de données vectorielles, renseignant sur les formations végétales en 1996 pour l'un, en 2011 pour l'autre.

Tableau 1. Inventaire des principales sources spatialisées, distinguées par nature et par période

\begin{tabular}{|c|c|c|c|c|c|c|c|}
\hline Périodes & Non daté & $\mathbf{V}^{\mathbf{e}}$ & $\mathbf{X V I}^{\mathbf{e}}$ & $\mathbf{X V I I}^{\mathbf{e}}$ & $\mathbf{X V I I}^{\mathbf{e}}$ & $\mathbf{X I X}^{\mathbf{e}}$ & $\mathbf{X X}^{\mathbf{e}}-\mathbf{X X \mathbf { I } ^ { \mathbf { e } }}$ \\
\hline Cartes et plans & $12^{*}$ & 1 & 1 & 4 & $9^{* *}$ & $13^{* * *}$ & 8 \\
\hline Photographies aériennes & \multicolumn{10}{|c|}{ Sans objet } & A partir de 1955: 10 séries \\
\hline & \multicolumn{10}{c}{ Sans objet } & & $\begin{array}{l}\text { - DNC 1996 et 2011, } \\
\text { - BD Forêt 2002 et 2011, } \\
\text { - BD Topo 2008 et 2011 }\end{array}$ \\
\hline
\end{tabular}

*Sur une partie du DNC parfois seulement : le château

** Dont carte de Cassini 1740

*** Dont cadastre napoléonien 1826 et carte d'état-major 1839

BD : Base de données ; DNC : Domaine national de Chambord

15 Les sources, bien que nombreuses, n'en présentent pas moins des limites, que nous allons discuter plus spécifiquement pour notre terrain d'étude, notamment en termes de disponibilité aux dates souhaitées, de pertinence et de fiabilité.

\section{Caractérisation des paysages : arbitrage entre dates, pertinence et fiabilité des sources}

Dans la phase de confrontation avec les sources, deux contraintes sont intervenues et ont dû être intégrées conjointement dans la démarche pour le choix définitif des dates clés ; il s'agit de la pertinence et de la fiabilité des sources. La question se pose souvent avec une plus grande acuité pour les dates les plus anciennes, impliquant de réduire, du moins d'adapter le choix des dates clés réalisé a priori (Robert, 2011), ce que nous allons voir à travers quelques exemples, en privilégiant les sources spécifiques au Domaine national de Chambord ${ }^{8}$.

\subsection{Du XVI e siècle à 1949 , les dynamiques de la forêt}

Pour mesurer l'impact du premier événement sur la couverture forestière - le début de la construction du mur entourant le domaine en 1524 (figure 1) -, deux états des lieux sont nécessaires, l'un avant, l'autre après 1524. Pour le premier, nous disposons d'une carte, délimitant le massif forestier au-delà de l'actuel domaine, extraite de l'ouvrage de J. Thoreau (1975) et qui daterait du début du Moyen Âge (source inconnue). Pour le second état des lieux, après 1524, plusieurs sources sont disponibles : deux cartes du Pays blaisois, celle de J. du Temps de 1591 et une autre datant de 1663, dressée après que le périmètre actuel du domaine a été arrêté (figure 1) ; une peinture murale de L. Poisson datant de 1600 et exposée au château de Fontainebleau. L'avantage de cette dernière est qu'elle offre davantage de détails, puisqu'elle est centrée sur le Domaine national de Chambord. Même si sa fiabilité peut être questionnée, dans la mesure où il s'agit d'une représentation artistique, elle nous a permis de réaliser une première cartographie de la forêt sur une grande partie du domaine (figure 2). On perçoit ainsi que cette formation 
végétale ne couvre pas l'ensemble du territoire et se concentre dans la partie méridionale. La confrontation avec d'autres états des lieux ultérieurs, en l'occurrence celui de 1810 (figure 3), nous confirme la fiabilité de cette source et nous conforte dans son choix.

Figure 2.1600, une première carte de la forêt du Domaine national de Chambord

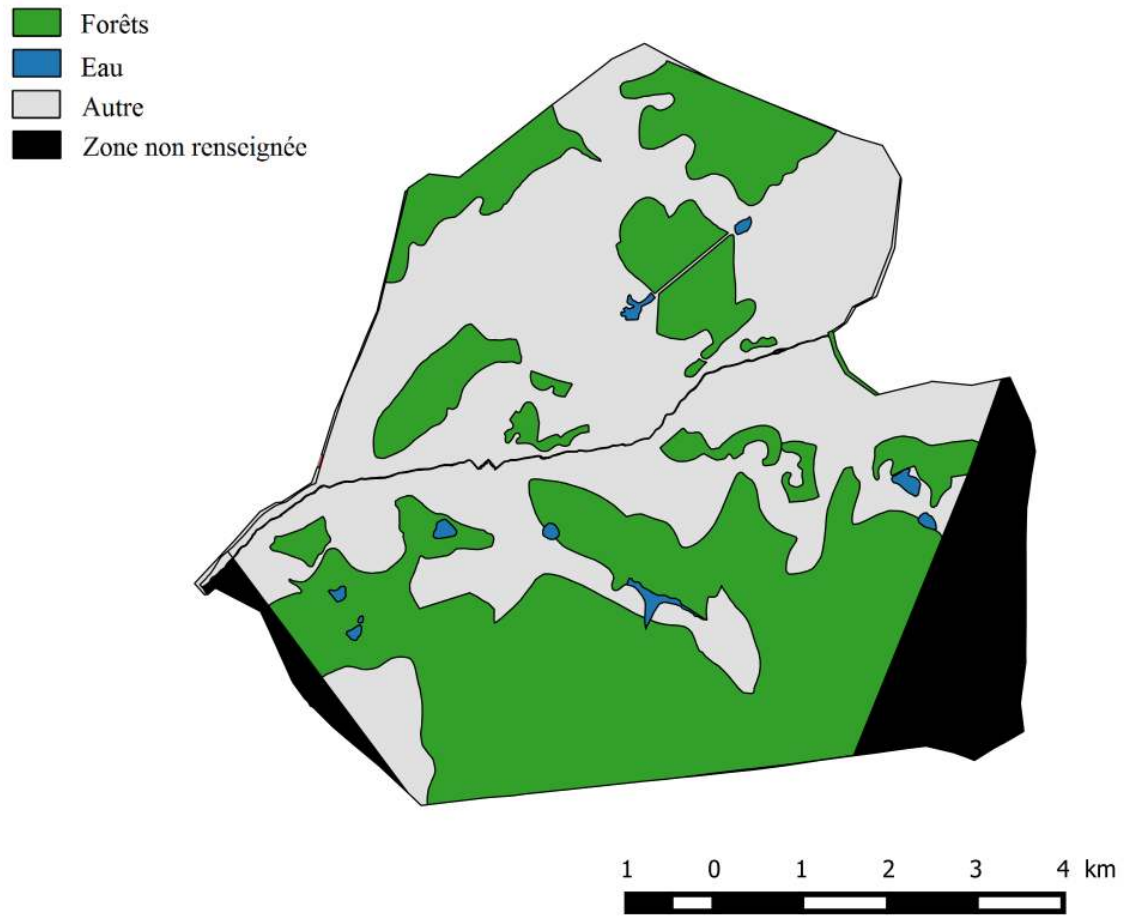

Autre : catégories non identifiées sur la source

Source : d'après la peinture murale de L. Poisson (exposée au château de Fontainebleau), 1600. Carte réalisée avec le logiciel QGIS

Un autre événement impose d'identifier les dynamiques d'occupation des sols, en dressant un état des lieux avant et après sa survenue ; ce sont les premiers reboisements réalisés en 1821. Une carte extraite de l'ouvrage de J. Thoreau (1975) est disponible à cette date, mais on peut douter de la concordance entre les informations représentées et la date, dans la mesure où elle est identique au plan géométral de la principauté de Wagram de 1810 (réalisé en 1846). La lecture de cette dernière s'avère complexe, mais elle permet de dresser un état des lieux avant reboisement, où sont notamment distinguées les forêts et les landes (figure 3). Les forêts ne couvrent qu'une partie du domaine, tandis que les landes constituent une catégorie d'occupation des sols tout aussi importante. Bien qu'un peu plus ancienne (1785), une autre source peut être mobilisée, d'autant plus qu'elle est plus complète, plus précise et plus facilement lisible (figure 4). Il s'agit du plan général du parc de Chambord au xviII ${ }^{\mathrm{e}}$ siècle (disponible au Centre historique des Archives nationales, NII Loir-et-Cher 2). Bien qu'il représente un projet, sa fiabilité concernant la superficie forestière est vérifiée par confrontation avec le plan de 1810 (figure 3). La carte, établie à partir de cette source, nous renseigne alors sur l'occupation des sols et nous révèle l'importance des terres agricoles à cette époque, s'étendant autour de plusieurs fermes. 
Figure 3. Carte des formations végétales du Domaine national de Chambord en 1810

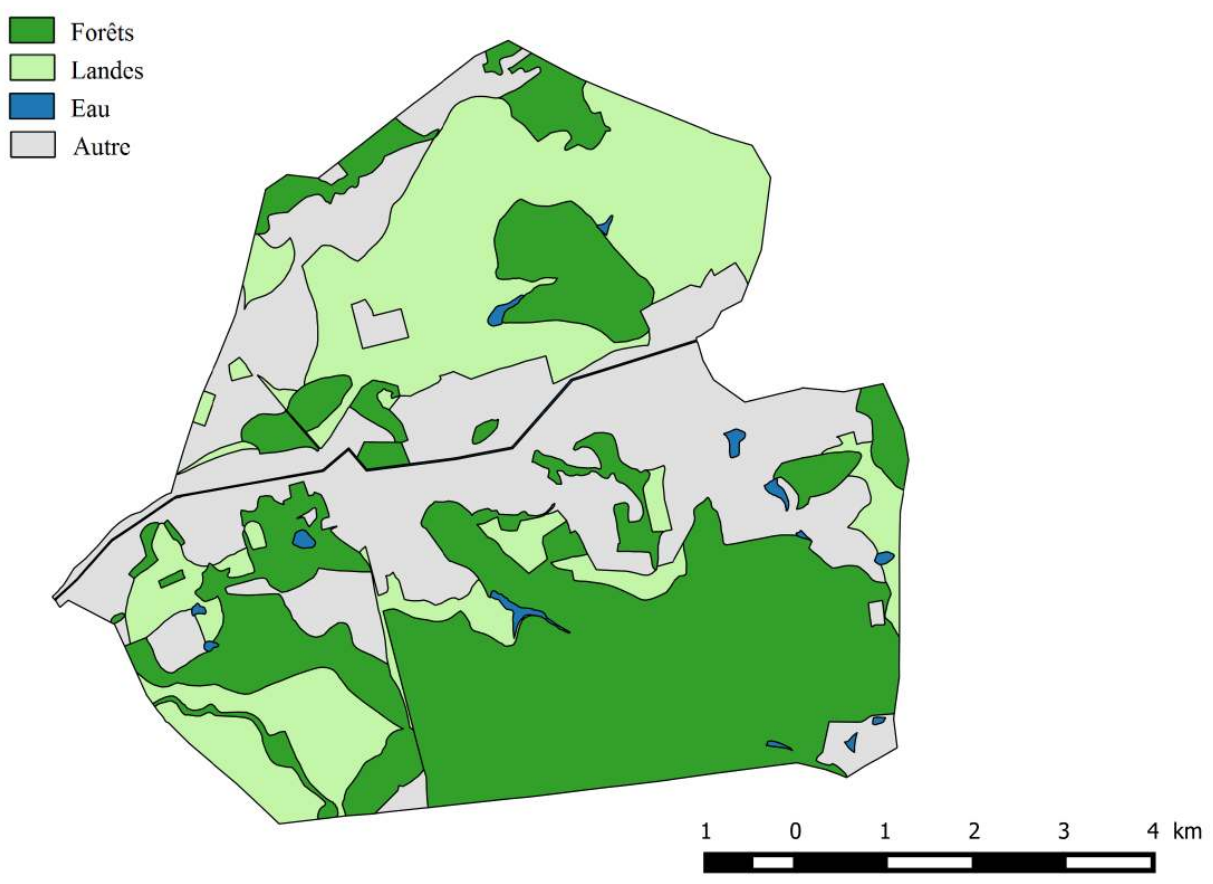

Source : d'après le plan géométral de la principauté de Wagram de 1810 (réalisé en 1846). Carte réalisée avec le logiciel QGIS.

Figure 4. Carte de l'occupation des sols du Domaine national de Chambord en 1785

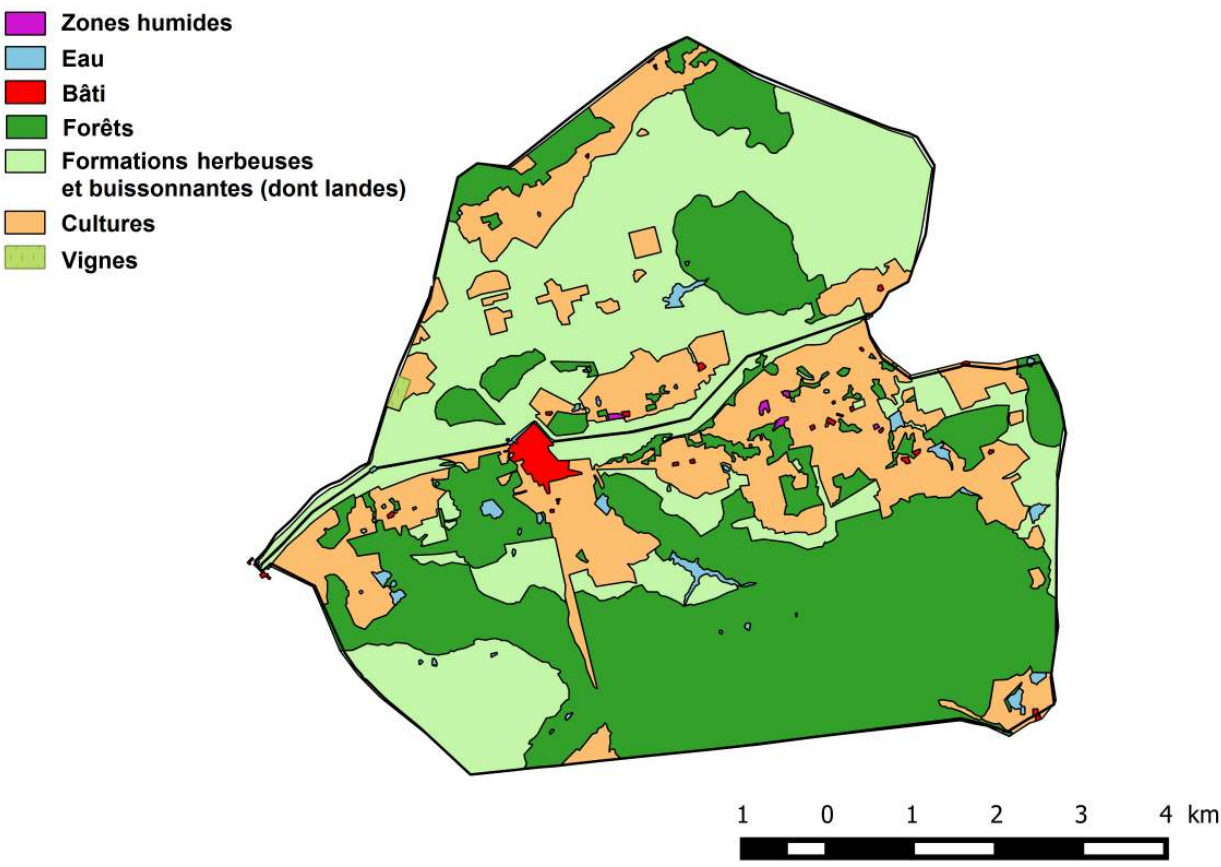

Source : d'après le plan général du Parc de Chambord, XVIII siècle (Centre historique des Archives nationales, NII Loir-et-Cher 2). Carte réalisée avec le logiciel QGIS 
On voit, à travers cet exemple, que la source dont la date est la plus proche de la date clé n'est pas nécessairement la plus pertinente et qu'il faut bien souvent procéder à un arbitrage entre la date, la pertinence et la fiabilité des sources. Par ailleurs, les sources les plus anciennes ne sont pas toujours les moins pertinentes, ce que démontre le plan de 1785. Par comparaison, pour l'état des lieux post-reboisements, deux sources aux typologies moins détaillées sont disponibles. L'une d'elles est un plan de 1857, trop schématique. L'autre serait plus satisfaisante, car elle apparaît moins simplifiée (Plan du parc de Chambord de N. Desmadryl, 1835, disponible à la Bibliothèque nationale de France) et deux types de formations végétales y sont aussi distingués, les forêts et les landes. Mais, par comparaison avec les états des lieux antérieurs (1785 et 1810), il semblerait que les reboisements ne soient pas pris en compte. La carte de 1857 (figure 5) serait alors plus pertinente pour un état des lieux post-reboisements puisque, en la comparant au plan de 1785 (figure 4), on perçoit bien la progression de la forêt. Pourtant, celle-ci semble exagérée pour la partie septentrionale du domaine, ne serait-ce qu'au vu des fermes demeurant en activité dans le domaine, avec la présence de terres agricoles omises sur la carte de 1857. En effet, J. Thoreau (1975) confirme que le nombre de fermes régresse, mais elles sont encore au nombre de six en 1947, s'étendant sur 482 hectares au total; on comprend là l'intérêt de se fonder aussi sur des données scripturales, afin de vérifier la fiabilité des sources cartographiques.

Les sources apparaissent relativement nombreuses pour retracer les dynamiques de cette forêt emmurée, mais leur fiabilité doit être questionnée. Surtout, peu de précisions sont données sur les peuplements, ce jusqu'en 1949 - ce qui justifie aussi de se focaliser sur la période post-1950, au-delà des objectifs du projet dans lequel s'inscrit notre étude -, même si les informations dont nous disposons suffisent à atteindre notre objectif d'identifier la place de la forêt dans le domaine et son évolution. 


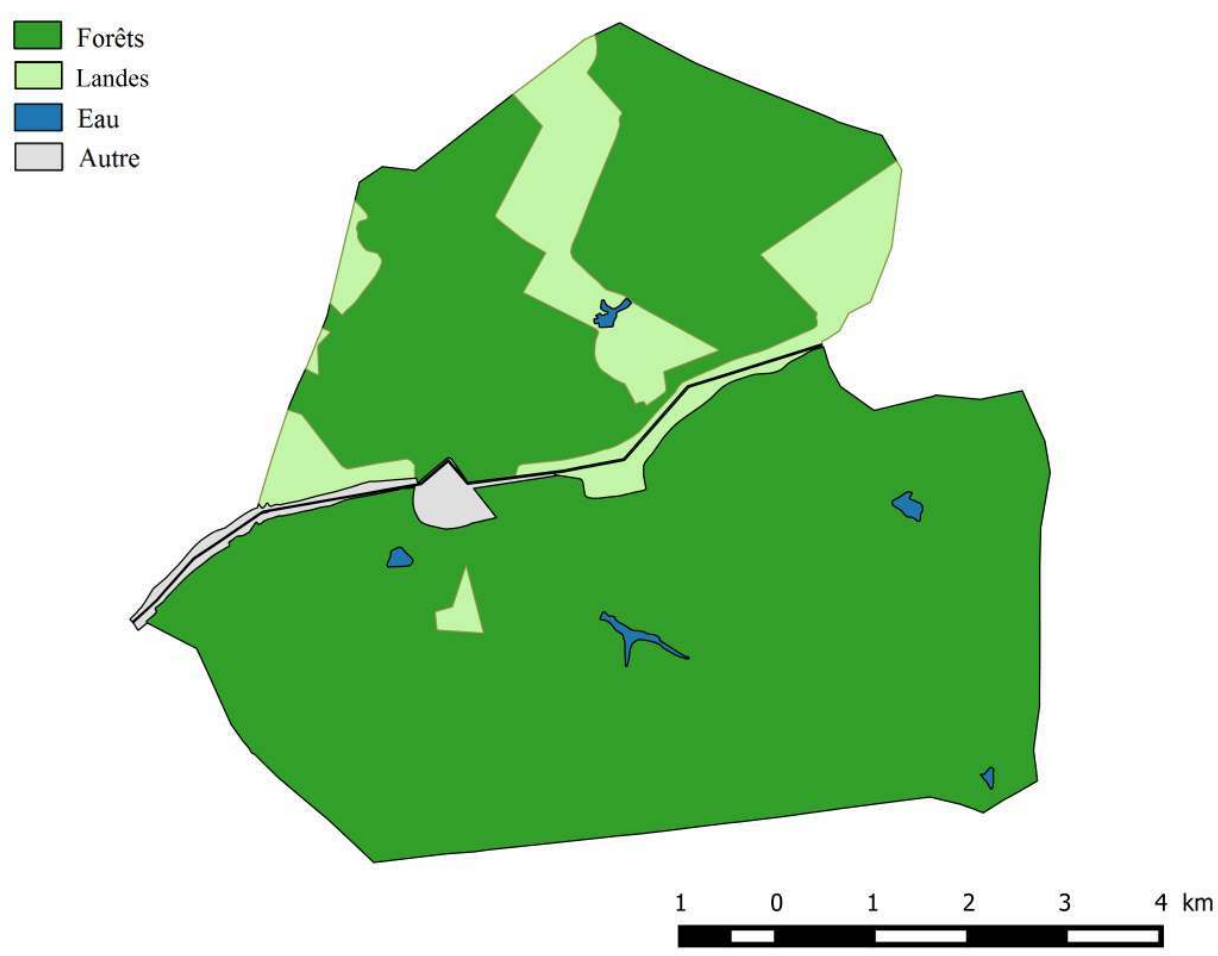

Source : d'après le plan du Domaine de Chambord, dressé par L. Marcellier, sous la direction de M. Bourcier, publié par A. Gatineau à Orléans, 1857. Carte réalisée avec le logiciel QGIS

\subsection{De 1949 à aujourd'hui, les dynamiques des peuplements forestiers}

Les sources les plus récentes sont plus riches et offrent alors une connaissance beaucoup plus précise de la forêt de Chambord, au-delà de sa seule délimitation, en identifiant les types de peuplements.

En 1949, la première carte des peuplements du parc de Chambord est réalisée par J. Thoreau (disponible aux archives du Domaine national de Chambord), source de la figure 6, qui en révèle la précision et une partie des détails9. Sont ainsi distinguées les futaies des taillis sous futaie. On perçoit alors la prédominance des seconds, ce qui est à mettre en lien avec la fonction cynégétique de la forêt. En effet, «Les taillis, recépés à intervalles réguliers, généralement tous les dix ou quinze ans, offrent aux cervidés une alimentation variée et abondante (feuilles des arbres, ronces, etc.) 》 (Buridant, 2005). Cette carte de 1949 (figure 6), se focalisant sur les peuplements, ne renseigne malheureusement pas sur les autres catégories d'occupation des sols. 


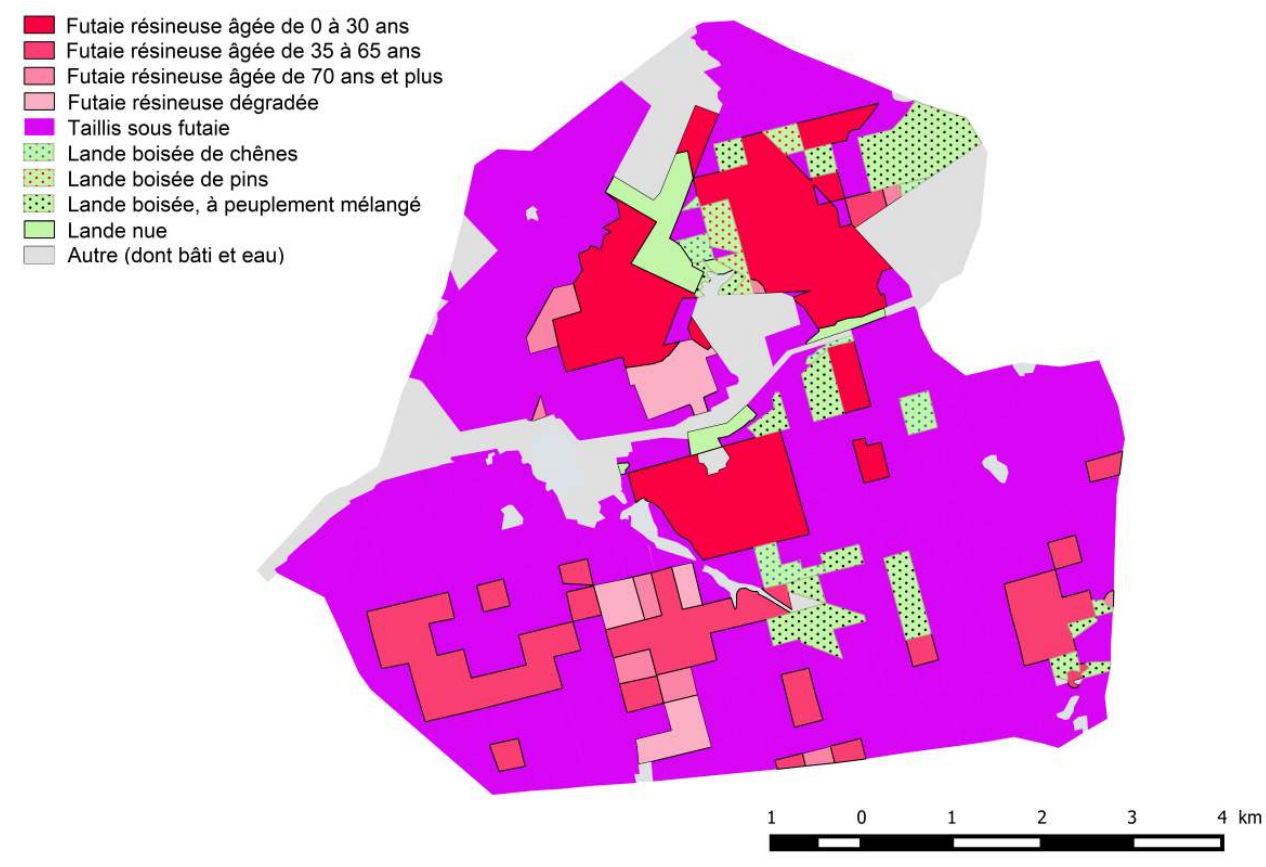

Source : d'après la carte des peuplements réalisée par J. Thoreau, mars 1949 (Archives du Domaine national de Chambord, 2011/1/321). Carte réalisée avec le logiciel QGIS

Pour l'état des lieux le plus récent, nous disposons de bases de données vectorielles qui renseignent sur les peuplements, de sorte qu'on est en mesure d'en identifier les évolutions, par comparaison avec l'état des lieux de 1949. Ces bases de données (BD) dressent la situation en 2011. Elles émanent de deux sources, le Domaine national de Chambord et l'IGN, chacune avec ses avantages et inconvénients, fonction de leurs objectifs distincts. La BD du Domaine national de Chambord se fonde sur des relevés de terrain précis, consistant en des inventaires de végétation systématiques, réalisés par les forestiers sur l'ensemble du domaine; l'objectif était la réalisation du plan d'aménagement de ce dernier. Cette source présente ainsi une très grande précision sur les formations végétales (figure 7-A), tout en renseignant aussi sur les autres catégories d'occupation des sols (figure 7-B). Il faut souligner que son utilisation a nécessité des corrections préalables, notamment en raison de la présence de polygones non jointifs. La $\mathrm{BD}$ Forêt de l'IGN offre également une cartographie des formations végétales. Établie à l'échelle nationale, elle se fonde sur des relevés moins fréquents et offre une moindre précision, mais, contrairement à toutes les autres sources mentionnées jusqu'à présent, qui sont spécifiques au Domaine national de Chambord, elle couvre une plus vaste surface et permet ainsi de resituer le domaine dans son environnement ${ }^{10}$. Au-delà de la plus faible précision spatiale, l'inconvénient est aussi que cette source ne renseigne que sur les formations végétales et, pour connaître les autres catégories d'occupation des sols, il faut faire appel à des sources complémentaires comme la BD Topo. 
Figure 7. Cartes de l'occupation des sols en 2011, d'après la BD du Domaine national de Chambord A. Les formations végétales

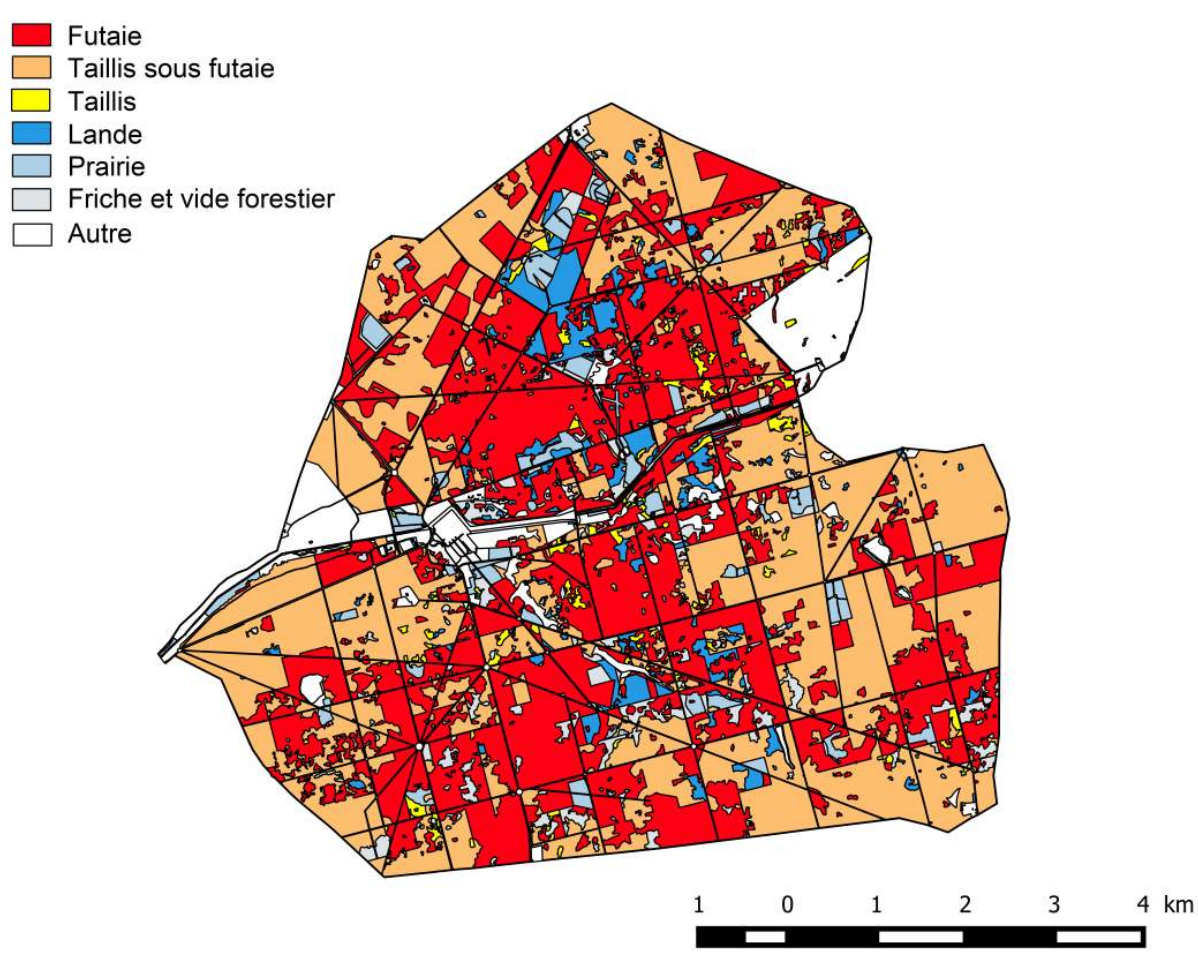

B. Toutes catégories d'occupation des sols

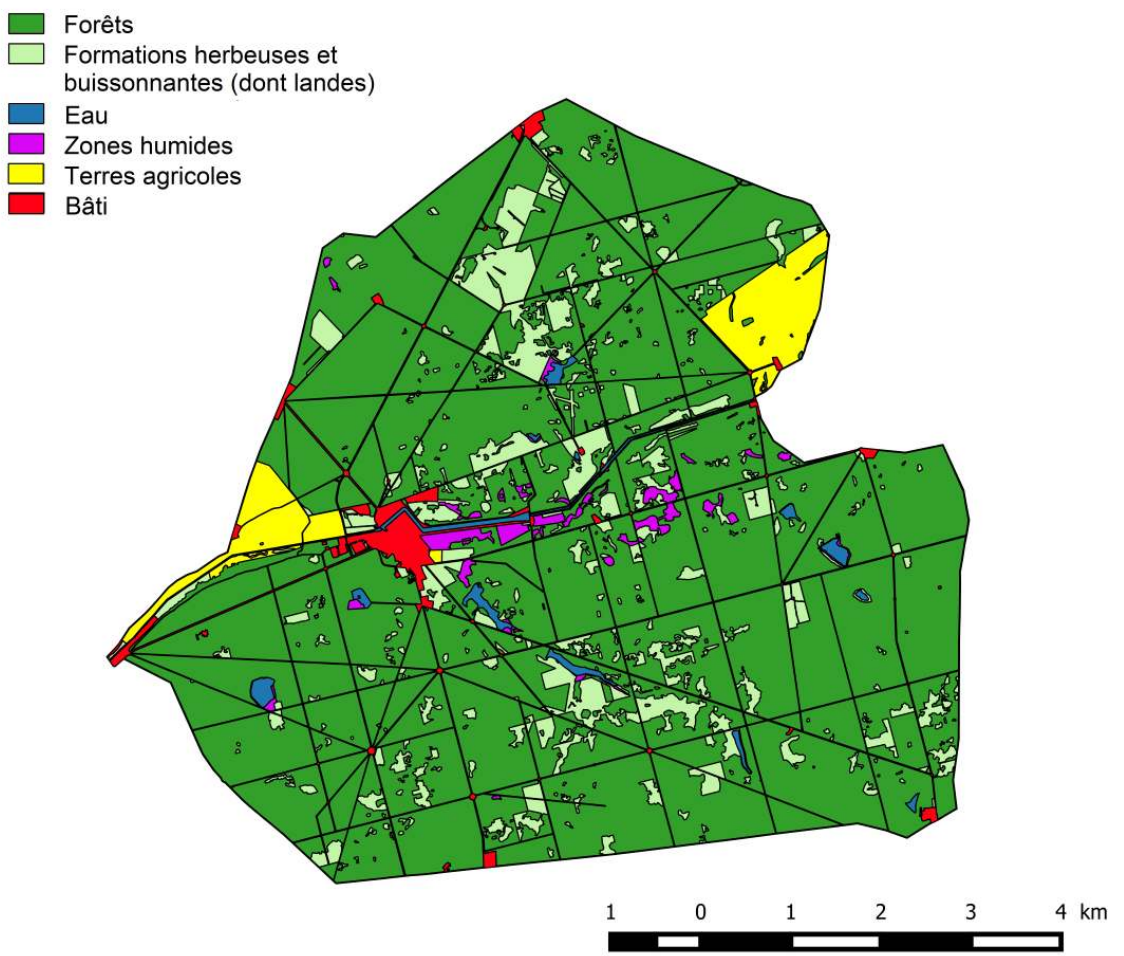

Source : d'après la BD du Domaine national de Chambord, 2011. Cartes réalisées avec le logiciel QGIS 
aucune source n'est satisfaisante, notamment parce qu'elles ne révèlent qu'une information parcellaire, ayant évidemment été réalisées en fonction d'objectifs qui ne sont pas ceux pour lesquels nous les mobilisons (Valette et Carozza, 2010), à savoir retracer les dynamiques forestières. Pour autant, dans le cas du Domaine national de Chambord, plus que pour d'autres terrains, d'autant plus si on songe à des terrains situés dans des pays des Sud où les données sont plus encore lacunaires (cas du Việt Nam, Robert, 2010 et 2011), ces sources permettent d'avoir une connaissance générale des dynamiques à l'œuvre depuis le XVII ${ }^{\mathrm{e}}$ siècle et d'identifier notamment l'événement le plus significatif: les reboisements $d u \mathrm{XIX}^{\mathrm{e}}$ siècle. Mais pour percevoir plus en détail les changements, il importe de confronter spatialement les différentes sources qui ont finalement été retenues, ce qui implique dès lors de mettre en place un SIG : on est ainsi en mesure de mener une analyse diachronique précise, en comparant les états des lieux issus des sources retenues. Ces dernières peuvent-elles toutes être intégrées dans un SIG ? D'autres sources, secondaires, peuvent-elles être mobilisées pour compléter la connaissance des paysages forestiers et de leurs dynamiques?

\section{L'identification des dynamiques paysagères : confrontation dans un SIG et mobilisation d'autres sources}

27 L'intérêt de confronter toutes les sources sélectionnées dans un SIG est de pouvoir identifier précisément les dynamiques d'occupation des sols ; cet outil est utilisé par de nombreux chercheurs menant de telles études géohistoriques (Amat et Hotyat, 1980 ; Amat, 1987 ; Houet et al., 2008 ; Franchomme et Dubois, 2010 ; Robert, 2011 ; ServainCourant et Yengué, 2011 ; Pin et al., 2013) et "l'importance du SIG dans l'identification des dynamiques de l'occupation du sol » (Carreau et Yengué, 2007) est désormais admise. Mais ceci n'est pas sans poser de difficultés et, pour certaines sources, il faut parfois même y renoncer.

\subsection{Les contraintes du SIG}

L'intégration de cartes et de plans dans le SIG nécessite un géoréférencement précis des sources retenues parfois complexe à réaliser ${ }^{11}$. Cette difficulté est particulièrement apparue pour la peinture de L. Poisson, qui a permis de dresser la carte de 1600 (figure 2) car, en tant que représentation picturale, elle ne respecte pas nécessairement la géométrie de l'espace ni la localisation des éléments qui y sont identifiés ${ }^{12}$. Le problème existe aussi pour d'autres sources, entraînant des décalages entre elles qui peuvent compromettre la connaissance des dynamiques spatiales. On s'attache bien sûr à réduire ce biais lors du géoréférencement, mais il importe de rester conscient de ces limites et 
d'intégrer une marge d'erreur possible dans la phase d'identification des permanences et mutations.

Pour connaître les dynamiques, les sources doivent être confrontées, mises en parallèle, ceci à partir de bases communes, ce qui implique alors une typologie identique, des catégories d'occupation des sols similaires. Il s'agit là de la seconde difficulté à laquelle on se heurte. Il importe alors de définir une typologie emboîtée, allant du plus précis au plus simple pour permettre la comparaison sur l'ensemble de la période analysée, du XVII siècle à aujourd'hui, sans que cela compromette notre objectif qui est d'identifier la place occupée par la forêt au cours de l'histoire. Ainsi, pour la comparaison entre 1949 (figure 6) et 2011 (figure 7), les types de peuplements sont considérés, mais pas les autres catégories d'occupation du sol, qui ne sont pas connues pour 1949. Lorsqu'il s'agit de retracer les dynamiques sur l'ensemble de la période étudiée, depuis le XVII ${ }^{\mathrm{e}}$, les cartes de 1949 et 2011 doivent être plus encore simplifiées pour pouvoir être comparées à des cartes plus anciennes. On distingue alors uniquement la forêt des autres classes d'occupation des sols. Il faut, à ce sujet, souligner que cette simplification ne concerne pas que les états des lieux récents, mais également de plus anciens, comme celui de 1785 . Dans le cadre de l'identification des dynamiques d'occupation des sols, elle n'empêche toutefois pas de compléter ponctuellement, de manière statique, l'analyse pour les dates où de plus amples informations sont disponibles. Plus encore, elles peuvent même en elles-mêmes renseigner sur ces dynamiques : tel est le cas de la carte de 1949 par exemple qui, par l'âge des peuplements, inclut en elle-même des précisions sur les dynamiques d'origine interne de la végétation.

On peut aussi envisager de prendre en compte d'autres sources, en l'occurrence iconographiques, qui, elles, ne seront pas nécessairement intégrables dans un SIG - ou sinon ponctuellement par la localisation du point d'origine de la prise de vue par exemple -, mais qui apporteront des informations complémentaires sur l'état de la forêt à un instant donné et, par comparaison globale, mais non moins spatialisée, sur ses dynamiques.

\subsection{Le recours à des sources complémentaires iconographiques}

31 Les sources complémentaires mobilisables, susceptibles de renseigner sur les dynamiques paysagères, sont notamment les sources iconographiques, les photographies, les gravures ou les peintures; elles complètent la connaissance plus partielle (en raison des limites inhérentes aux sources) des dynamiques passées. Les premières offrent des fenêtres sur les paysages, mais qui peuvent être orientées (Ertlen et Schwartz, 2010 ; Robert, 2011). De la même manière, les gravures et les peintures révèlent les paysages tels que leurs auteurs se les représentent ou tels qu'ils souhaitent les montrer, d'un point de vue surtout esthétique, ce qui ne correspond pas nécessairement aux paysages réels. On pourra alors considérer que ces sources sont en partie subjectives et qu'elles sont dès lors biaisées au regard de nos objectifs. Pour autant, elles révèlent les paysages perçus par leurs auteurs et, à travers eux, par la société de l'époque. Par ailleurs, nous interprétons aussi nous-mêmes inévitablement les paysages par le prisme de nos propres représentations (Jacob-Rousseau, 2009).

Au-delà, ces sources iconographiques peuvent compléter la connaissance des dynamiques, en offrant un aperçu des paysages entre deux états des lieux. Elles sont alors confrontées aux sources cartographiques, ce qui permet d'en vérifier la fiabilité. Les 
sources iconographiques peuvent confirmer aussi des hypothèses apparues au regard de l'analyse des données cartographiques. Ainsi, une gravure du château de Chambord, réalisée par L.-A. Asselineau (Archives départementales du Loir-et-Cher, 88 Fi 5), confirme la présence de terres agricoles dans les années 1860 au sein du domaine, que nie la carte de 1857 (figure 5). La fiabilité des sources cartographiques autant qu'iconographiques doit être questionnée et vérifiée par leur comparaison, nous permettant d'obtenir des informations pertinentes sur la place occupée par la forêt au sein du domaine depuis sa création.

Les sources iconographiques du Domaine national de Chambord renseignent aussi sur les pratiques de l'époque, comme l'agriculture qui était relativement présente jusqu'au XIX ${ }^{\mathrm{e}}$ siècle, mais surtout la chasse. Cette dernière est ici fondamentale pour comprendre les dynamiques qui affectent la forêt de Chambord, façonnée pour devenir un domaine de chasse (Buridant, 2005). La pratique de la chasse explique ainsi la progression de la forêt dans le domaine, mais aussi la construction du mur qui en fait une "forêt emmurée " pour conserver le gibier sur ces terres et s'affranchir des contraintes juridiques (ibid.). Des aménagements spécifiques en découlent également : outre une organisation en étoile des voies de circulation, ce sont des modes de gestion appliqués aux forêts. Ainsi, les taillis sous futaie importent pour nourrir le gibier (ibid.) et c'est ce qui explique qu'ils sont majoritaires dans le domaine en 1949 (figure 6), même s'ils régressent ensuite, entre 1949 et 2011. La futaie est, elle, favorisée pour la chasse à courre, car elle «facilite la poursuite du gibier et permet de belles chevauchées » (ibid.), mais, dans le domaine, elle cesse en 1947, avec la création de la Réserve nationale de chasse et de faune sauvage. On perçoit là l'intérêt d'autant plus grand pour notre étude de prendre en compte les types de peuplements. La forêt du Domaine national de Chambord a ainsi été fortement marquée par la pratique de la chasse et elle le demeure aujourd'hui encore, même si d'autres activités tendent de plus en plus à façonner le domaine, engendrant de nouvelles dynamiques: elles sont à mettre en lien avec la volonté de ses gestionnaires d'inclure davantage le parc dans l'offre touristique qu'ils proposent.

\section{Conclusion}

La disponibilité des sources diffère selon les terrains et, même à l'échelle nationale, il existe des disparités ; certains terrains apparaissent plus documentés que d'autres. Tel est le cas du Domaine national de Chambord qui constitue un cas d'étude en la matière, un espace emmuré bien cartographié. Pour autant, même pour un tel espace, la reconstitution des dynamiques forestières se heurte aux limites inhérentes aux sources, qui se posent en termes de disponibilité, de fiabilité, de pertinence aussi, puisqu'elles ont été réalisées en fonction d'autres objectifs que ceux que nous nous sommes fixés. Elles se posent d'autant plus à mesure qu'on remonte le passé, mais sans disparaître pour la période plus récente, où certes les données sont plus riches, plus nombreuses, mais nécessitent parfois d'être corrigées (cas des bases de données vectorielles). Ces limites inhérentes aux sources contraignent alors la connaissance des temporalités, celles où se rencontrent les dynamiques spontanées de la forêt et celles impulsées par la société. Pour tenter d'y pallier, le recours à des sources hétérogènes, de natures diverses (cartographiques, mais aussi iconographiques, scripturales, orales ; Robert, 2010 et 2011) peut être envisagé. L'avantage est d'intégrer à l'analyse des dynamiques d'occupation des sols et des paysages des éléments sur les représentations de la société de l'époque, avec 
toutes les précautions d'interprétation que cela implique, nous l'avons vu. Cette approche méthodologique des temporalités que nous avons ici proposée, à travers l'exemple des paysages forestiers de Chambord et leurs évolutions, est une approche géographique, plus précisément biogéographique. Elle permet d'appréhender un «temps des territoires » où se mêlent des dynamiques spontanées, «naturelles » et des dynamiques sociétales aux effets perceptibles dans l'espace, à travers les paysages. L'objectif n'est alors pas de retracer les dynamiques pour elles-mêmes, mais plutôt pour comprendre les paysages actuels. Comme pour d'autres géographes, "à l'aide notamment de l'outil SIG, il s'agit [...] d'analyser lorsque c'est possible l'impact des pratiques anciennes sur les paysages actuels» (Rochel, 2016); de "mieux appréhender leur évolution et [ainsi] de les gérer au mieux» (Hotyat, 2012). Tel est l'objectif de notre étude, menée dans le cadre d'un programme de recherche qui ambitionne de proposer des solutions pour aboutir à une meilleure gestion des fortes populations d'ongulés sauvages et de leurs habitats. L'analyse se poursuit, sur les dynamiques et leurs facteurs surtout anthropiques, mais nos travaux démontrent déjà que l'événement majeur qu'a connu le domaine fut les plantations du XIx ${ }^{\mathrm{e}}$ siècle et que la chasse marque les paysages, même si de nouveaux usages récréatifs apparaissent aujourd'hui.

\section{BIBLIOGRAPHIE}

Amat J.-P., 2015, Les forêts de la Grande Guerre. Histoire, mémoire, patrimoine, Paris, Presses de l'université Paris-Sorbonne, $548 \mathrm{p}$.

Amat J.-P., 1987, « Guerre et milieux naturels : les forêts meurtries de l'est de la France, 70 ans après Verdun », L'espace géographique, vol. 16, nº 3, p. 217-233.

Amat J.-P., Hotyat M., 1980, « Approche méthodologique biogéographique et géomorphologique de la platière d'Apremont ", Annales de géographie, vol. 89, n 494, p. 490-495.

Baltzinger C., 2016, « Pour une approche intégrée du rôle des ongulés sauvages dans l'assemblage des communautés végétales et le fonctionnement des écosystèmes ", habilitation à diriger des recherches en sciences de la vie - Biologie des populations et écologie, université d'Orléans, $138 \mathrm{p}$.

Buridant J., 2005, « La gestion des forêts de vénerie au XVII ${ }^{\mathrm{e}}$ siècle ", dans XVII siècle, « Chasse et forêt ", 2005/1 ( $\left.{ }^{\circ} 226\right)$, Presses universitaires de France, p. 17-27.

Carreau C., Yengué J. L. (2007), « Approche méthodologique pour l'étude des dynamiques paysagères. Le cas de l'Indre et Loire », Conférence francophone ESRI SIG 2007, octobre, Versailles, 15 p., http://www.esrifrance.fr/sig2007/univ_tours.htm, consulté le 12/12/2015.

Carrelet-Baltzinger M., 2016, Political ecology des engrillagements de Sologne - Tentative de défragmentation du paysage écologique, politique et disciplinaire, thèse de doctorat, Agrosystèmes, écosystèmes et environnement, sous la direction de M. Deconchat et A. Marell, université de Toulouse, $359 \mathrm{p}$. 
Côté S. D., Rooney T. P., Tremblay J.-P., Dussault C., Waller, D. M.., 2004, « Ecological impacts of deer overabundance ", Annual Review of Ecology Evolution and Systematics, vol. 35, p. 113-147.

Dubar C., Rolle, C., 2008, « Les temporalités dans les sciences sociales : introduction », Temporalités , 8, 2008, http://temporalites.revues.org/57, mis en ligne le 09/07/2009, consulté le 30/08/2016.

Ertlen D., Schwartz D., 2010, « Utilisation d'archives géohistoriques pour la mise au point d'une nouvelle méthode paléoécologique ", Géocarrefour, vol. 85/1, mis en ligne le 27/05/2010, http:// geocarrefour.revues.org/7695, consulté le 21/04/2017 ; DOI : 10.4000/geocarrefour.7695.

Franchomme M., Dubois J.-J., 2010, « Documenter les zones humides : vers une meilleure compréhension des paysages de l'eau du XIX ${ }^{\mathrm{e}}$ au XX $\mathrm{Xx}^{\mathrm{e}}$ siècle », Géocarrefour, vol. 85/1, http:// geocarrefour.revues.org/7622, consulté le 21/04/2017.

Galochet M., Glon E., 2010, « Des milieux aux territoires forestiers : itinéraires biogéographiques et postures scientifiques », in Galochet M. et Glon E. (dir.), Des milieux aux territoires forestiers Mélanges en l'honneur de Jean-Jacques Dubois, Artois Presses université, collection « Géographie », p. 33-47.

Georges-Leroy M. et al., 2011, « Apport du LiDAR à la connaissance de l'histoire de l'occupation du sol en forêt de Haye ", ArcheoSciences, ${ }^{\circ} 35$, mis en ligne le 30/04/2013, http:// archeosciences.revues.org/3015, consulté le 23/04/2017; DOI : 10.4000/archeosciences.3015.

Horen H., Buridant J., Gallet-Moron E., Brasseur B., Feiss T., Heraude M., Rassat S., Montoya C., Burban-Col V., 2015, « Relation entre les structures archéologiques identifiées sur un levé LiDAR et la typologie des sols du massif forestier de Compiègne (nord de la France) », Revue du Nord, "Sols en mouvement : rencontres internationales de Liessies 2014 », université Charles-deGaulle, Lille 3.

Hotyat M., 2012, « Intérêt de l'approche systémique pour l'étude de formations végétales en milieux forestiers : l'exemple de la forêt de Fontainebleau », Vertigo, hors-série $n^{\circ} 14$, septembre, http://vertigo.revues.org/12446, consulté le 12/12/2015.

Houet T., Hubert-Moy L., Corgne S., Marchand J.-P., 2008, « Approche systémique du fonctionnement d'un territoire agricole bocager », L'espace géographique, $\mathrm{n}^{\circ} 3$ (tome 37), p. 270-286.

Jacob-Rousseau N., 2010, « Des archives aux paysages : milieux, dynamiques, territoires », Géocarrefour, vol. 85/1, p. 3-6, http://geocarrefour.revues.org/7619, consulté le 26/12/2015.

Jacob-Rousseau N., 2009, « Géohistoire/géo-histoire : quelles méthodes pour quel récit ?», Géocarrefour, vol. 84/4, http://geocarrefour.revues.org/7598, consulté le 21/04/2017.

Lepart J., Marty, P., Klesczewski M., 2007, « Chapitre 2 - Faut-il prendre au sérieux les effets des changements du paysage sur la biodiversité ? ", in Berlan-Darqué M. et al., Paysages : de la connaissance à l'action, Éditions Quæ, collection « Update Sciences \& Technologies », p. 29-40.

Mårell A., Pellerin M., Rocquencourt A., 2012, « Mesure et suivi de l'empreinte écologique des ongulés sauvages sur la végétation forestière », Revue forestière française, n 5-2012, p. 711-721.

Matos Machado (de) R., Amat J.-P., Arnaud-Fassetta G., Bétard F., 2016, « Potentialités de l'outil LiDAR pour cartographier les vestiges de la Grande Guerre en milieu intra-forestier (bois des Caures, forêt domaniale de Verdun, Meuse) ", ÉchoGéo, n 38, mis en ligne le 13/12/2016, http:// echogeo.revues.org/14791, consulté le 23/04/2017 ; DOI : 10.4000/echogeo.14791.

Pin B., Rode S., Servain S., 2013, « Processus de construction d'une ressource territoriale valorisant le patrimoine naturel et culturel en Loire tourangelle », Vertigo, hors-série $n^{\circ} 16$, juin, http://vertigo.revues.org/13713, consulté le 22/12/2015 ; DOI : 10.4000/vertigo.13713. 
Robert A., 2011, Dynamiques paysagères et guerre dans la province de Thừa Thiên Huế (Việt Nam central), 1954-2007 - Entre défoliation, déforestation et reconquêtes végétales, thèse de doctorat, géographie, sous la direction de J.-P. Amat, université Paris-Sorbonne, 1172 p. + Atlas (159 p.)

Robert A., 2010, «Reconstitution de la forêt de la province vietnamienne de Thừa Thiên Huế durant un siècle tourmenté ", in Galochet M. et Glon E. (dir.), Des milieux aux territoires forestiers Mélanges en l'honneur de Jean-Jacques Dubois, Artois Presses université, collection « Géographie », p. 119-137.

Rochel X., 2016, « Paysages naturels, paysages construits. Géographie historique et usage des archives dans l'étude des paysages dits naturels (forêts, saltus) », habilitation à diriger des recherches en géographie, vol. 1 (texte inédit), université de Lorraine, 210 p.

Rochel X., 2013, « Sources d'archives et histoire de l'environnement. L'origine des hêtraies vosgiennes d'après les registres de martelages du XVIII ${ }^{\mathrm{e}}$ siècle ", in Galop D. (dir.) Paysages et environnement. De la reconstitution du passé aux modèles prospectifs, Besançon, Presses universitaires de Franche-Comté, Annales littéraires 909, série « Environnement, sociétés et archéologie » vol. 16, p. 15-24.

Servain-Courant S., Yengué J. L., 2011, « Les forêts du Val de Loire, maillons dans un paysage emblématique : Bréhémont en Indre-et-Loire ", in Corvol A. (dir.), Forêt et paysage. $X^{e^{-}} \mathrm{XXI}^{e}$ siècle, Paris, L'Harmattan, p. 131-140.

Thoreau J., 1975, Chambord rendez-vous de chasse, Paris, Librairie des Champs-Élysées, 288 p.

Valette P., Carozza J.-M., 2010, « Mise en œuvre d'une démarche géohistorique pour la connaissance de l'évolution des paysages fluviaux : l'exemple de la moyenne vallée de la Garonne ", Géocarrefour, vol. 85/1, http://geocarrefour.revues.org/7679, consulté le 21/04/2017.

\section{NOTES}

1. Au sein du domaine s'ajoutent des engrillagements, qui remplissent une autre fonction, plutôt défensive. Ils visent en effet à protéger les habitations, le château ou les zones de régénération forestière des dégâts imputables aux ongulés sauvages.

2. Ce projet de recherche s'intitule «Contribution des Ongulés au foncTionnement de l'écosystème et AUx services rendus à ChamborD » (Costaud). Financé par la Région Centre-Val de Loire, il est porté par l'Irstea et il s'inscrit dans le chantier «Chambord » d'Intelligence des Patrimoines - programme soutenu par la Région Centre-Val de Loire, animant un réseau de chercheurs en partenariat avec les mondes culturels et économiques.

3. Dans le cadre de notre projet de recherche sont aussi menées des études sur les caractéristiques des sols et sur la diversité végétale à l'échelle de placettes de relevés. L'objectif de notre travail est alors de renseigner aussi sur l'évolution de l'occupation des sols sur les parcelles considérées. On pense notamment à la continuité de l'état boisé (notion de forêt ancienne) sur le fonctionnement des sols et la flore associée.

4. D'autres travaux de recherche sont réalisés par le Laboratoire d'archéologie de Tours (UMR CITERES), afin de reconstituer les paysages sur un temps plus long encore, en se fondant sur des images LiDAR (projet financé par la Région Centre-Val de Loire, SOLiDAR: «Diachronie de l'occupation du sol : télédétection LiDAR en forêt de Chambord, Russy, Boulogne et Blois ", dirigé par X. Rodier).

5. Lepart J. et al., 1996, «Photographie diachronique et changement des paysages. Un siècle de dynamique naturelle de la forêt à Saint-Bauzille-de-Putois, vallée de l'Hérault ", Forêt méditerranéenne, $\mathrm{n}^{\circ} 17$, p. 63-80. 
6. Debussche M. et al., 1999, « Mediterranean landscapes changes: evidence from old postcards ", Global Ecology and Biogeography, $n^{\circ}$ 8, p. 3-15.

7. « Globalement la qualité des documents s'accroît avec le temps » (Valette et Carozza, 2010), même si certaines périodes peuvent s'avérer fécondes et offrir des sources cartographiques de plus grande qualité. Tel est le cas par exemple en Lorraine au XVIII ${ }^{\mathrm{e}}$ siècle, où « les plans paraissent fiables» (Rochel, 2016). De même, la cartographie historique actuelle, "renouvelée n'est peut-être pas toujours plus riche qu'il y a trente ou quarante ans " (ibid.).

8. Afin de faciliter l'analyse figurent dans cette section des cartes qui ont été dressées à partir des sources mentionnées.

9. La figure 6 est une version simplifiée de la carte d'origine de J. Thoreau. En effet, celle-ci distingue, pour les taillis sous futaie par exemple, les "taillis sous futaie à conserver", les " peuplement $[\mathrm{s}]$ complet $[\mathrm{s}]$, taillis rabougri $[\mathrm{s}]$ », les «peuplement $[\mathrm{s}]$ complet $[\mathrm{s}]$, réserves très denses ", les " réserves éparses, taillis éliminé[s]», les « peuplements mélangés, avec vieux pins à régénérer » et les " peuplements mélangés, feuillus, résineux ».

10. Il en est de même, à des dates plus anciennes, des cartes de Cassini (1740) et d'état-major (1839). Dans cet article, nous avons pris le parti de présenter des sources spécifiques au Domaine national de Chambord, plutôt que celles-ci qui sont davantage connues, d'autant plus qu'elles sont moins précises et, pour la carte de Cassini, sa date ne correspond pas aux dates clés que nous avons sélectionnées.

11. Dans notre cas, la BD vectorielle de 2011 du Domaine national de Chambord a servi de référence, dans la mesure où elle constitue le point de départ de notre analyse, correspondant à la source la plus récente pour connaître, qui plus est précisément, les paysages actuels. Plusieurs types de transformation ont été à chaque fois testés, avec l'objectif de limiter les décalages entre les données ; la polynomiale a souvent été préférée, sauf pour les photographies aériennes, où la linéaire présentait l'avantage d'éviter les déformations.

12. Le géoréférencement de cette peinture a été réalisé par le Domaine national de Chambord et vérifié par confrontation avec les autres sources.

\section{RÉSUMÉS}

Le Domaine national de Chambord est un espace emmuré bien documenté. Dans cet article, nous présentons la méthodologie mise en œuvre pour en retracer les dynamiques à partir du XVII siècle, afin de mieux comprendre les paysages actuels. Les dates clés et la nature des sources (spatialisées) sélectionnées sont précisées. Puis ces choix sont confrontés à la réalité des sources et ces dernières dévoilent alors leurs limites (disponibilité, fiabilité, pertinence). Leur analyse s'appuie sur un SIG, pour identifier les dynamiques, avec là aussi des implications, et elle doit s'enrichir par la mobilisation de sources complémentaires. Ainsi, même pour un tel espace, bien documenté, les limites inhérentes aux sources contraignent la connaissance des temporalités.

The national property of Chambord is a well-documented walled area. In this article, we present the methodology that we used to trace its dynamics from the XVII ${ }^{\text {th }}$ century to better understand the current landscapes. We first specify the key dates and the nature of the selected sources (the spatialized ones). Then these choices are confronted with the reality of the sources; we thus see the limits of these ones (availability, reliability, relevance). To identify the dynamics, the analysis of the selected sources is based on a GIS, which has some implications too, and the main sources 
have to be enriched by some complementary sources. Thus, even for such a space, which is welldocumented, the limits linked to the sources compel the knowledge of temporalities.

\section{INDEX}

Mots-clés : forêt, dynamiques, temporalités, sources, SIG, Chambord

Keywords : forest, dynamics, temporalities, sources, GIS, Chambord

\section{AUTEURS}

\section{AMÉLIE ROBERT}

Amélie Robert est docteur en géographie. Elle mène des recherches sur les paysages et les services écosystémiques, dans une perspective géohistorique, interrogeant plus généralement les interrelations entre les sociétés et leur environnement. Université de Tours, UMR CITERES. amelie.robert@univ-tours.fr

\section{SYLVIE SERVAIN}

Sylvie Servain est professeur des universités en géographie. Elle développe principalement des travaux de recherche sur les dynamiques des territoires et la construction paysagère des espaces fluviaux. INSA Centre Val de Loire et UMR CITERES. sylvie.servain@insa-cvl.fr 